\title{
The Interaction of REM Sleep with Safety Learning in Humans: Could a Good Night's Sleep Alter a Traumatic Experience?
}

\author{
-Rowan P. Ogeil ${ }^{1,2}$ and $\odot$ Kathryn D. Baker ${ }^{3}$ \\ ${ }^{1}$ Eastern Health Clinical School, Monash University, Box Hill 3128, Australia, ${ }^{2}$ Turning Point, Eastern Health, Fitzroy 3065, Australia, and ${ }^{3}$ School of \\ Psychology, The University of New South Wales, Sydney 2052, Australia \\ Review of Marshall et al.
}

Post-traumatic stress disorder (PTSD) is a heterogeneous anxiety disorder affecting $8 \%$ of the population (Briscione et al., 2014). It is characterized by hyperarousal, intrusive reminders of the traumatic event, avoidance of trauma-related cues, and negative cognition and mood (Briscione et al., 2014). There is increasing evidence that sleep disturbances, particularly in REM sleep, are important factors in the determination and progression of PTSD, and independently contribute to poor daytime functioning and treatment resistance in PTSD sufferers (Germain et al., 2013).

Animals subjected to fear conditioning and extinction training have provided robust models to understand the progression and underlying mechanisms of PTSD (Briscione et al., 2014). Studies examining fear learning typically use Pavlovian procedures, which involve pairing an initially neutral conditioned stimulus (CS) with a naturally aversive unconditioned stimulus (US). Fear responses to a CS can be extinguished by repeated presentation of the CS by itself. The extinction of learned

Received Oct. 21, 2014; revised Dec. 1, 2014; accepted Dec. 3, 2014.

R.P.O. and K.D.B. are supported by National Health and Medical Research Council Peter Doherty Early Career Fellowships (APP1071725 and APP1054642).

Correspondence should be addressed to Dr Rowan P. Ogeil, Turning Point, 54-62 Gertrude Street, Fitzroy, VIC, 3065, Australia. E-mail: Rowan.0gei@monash.edu.

DOI:10.1523/JNEUROSCI.4370-14.2015

Copyright $\odot 2015$ the authors $\quad 0270-6474 / 15 / 351337-03 \$ 15.00 / 0$ fear is the basis of exposure-based therapies, currently recognized as the gold standard treatment for anxiety disorders, including PTSD.

Although studies of fear conditioning in animals have focused primarily on dysregulation of fear processing, a central feature of PTSD, they are also useful in demonstrating how sleep can be disturbed after fear learning. Animal studies have consistently shown that REM sleep is disrupted after fear conditioning, and experimentally induced REM sleep disruption after fear conditioning impairs extinction learning the next day (Briscione et al., 2014). In addition, disruption of REM sleep during the consolidation period after extinction training impairs the retention of extinction (Fu et al., 2007; Datta and O'Malley, 2013). This suggests that REM sleep plays a role in PTSD via interactions with fear and extinction consolidation processes, but there has been limited study of these factors in humans.

In a recent issue of The Journal of $\mathrm{Neu}$ roscience, Marshall and colleagues (2014) provide a unique translational study that examines whether fear conditioning disrupts overnight REM sleep in humans. The authors hypothesized that greater levels of fear conditioning during the day would lead to higher fragmentation of REM sleep at night. The authors also examined the relationship between REM sleep and the generalization of fear to a safety signal. A safety signal is a stimulus (the $\mathrm{CS}-$ ) presented during fear learning but never paired with the aversive US. The examination of safety signals was of particular interest because impaired safety signal learning is observed in PTSD (Jovanovic et al., 2012). Marshall and colleagues (2014) hypothesized that greater levels of safety learning would be associated with more consolidated REM sleep.

Healthy participants $(n=42)$ were housed in a sleep laboratory for three consecutive nights, consisting of an adaptation night, a baseline night, and a testing/ outcome night. An advantage of this procedure was that a consistent sleepwake schedule was part of the inclusion criteria, and before the study, adherence to this schedule was monitored. Fear conditioning took place after the baseline night. In this session, a visual CS was paired with a shock to the wrist (CS+) and another visual CS was never paired with the shock (CS-). Learned fear and safety responses were measured using a fear-potentiated startle procedure, in which the measure of fear was the magnitude of an eye-blink startle response elicited by a brief auditory stimulus presented in the presence or absence of the CS. Participants were tested for retention the next day. Each night, polysomnography measures were recorded for 
participants, with three REM variables serving as primary outcome measures: (1) REM\%, (2) REM sleep efficiency, and (3) REM sleep latency. Lower or shorter measures of each of these represented REM fragmentation.

In contrast to their hypothesis, Marshall et al. (2014) reported that the strength of fear learning to the reinforced CS did not predict REM sleep fragmentation. The authors proposed that this could be due to the less aversive nature of the fear-potentiated startle procedure when compared with typical animal studies. Instead, REM sleep was more robustly related to safety signal learning and consolidation. Increased safety signal learning during the acquisition phase was associated with increased REM sleep consolidation later that night. Moreover, increased REM sleep consolidation was related to better discrimination of the safety and threat signals the following day.

These findings provide novel evidence linking safety learning and REM sleep in humans. In support of this link, there is an overlap in neural substrates underlying REM sleep and safety signal learning, providing a biologically plausible mechanism. Both REM sleep and safety signal learning are characterized by enhanced limbic system activity in the hippocampus and medial prefrontal cortex. Although specific neural mechanisms were not tested, Marshall et al. (2014) propose that REM sleep after safety learning may lead to increased neurogenesis within the hippocampus and/or medial prefrontal cortex, which, in turn, improves fear and safety discrimination the next day. However, increased neurogenesis in limbic regions would be expected to occur with other types of hippocampal-dependent learning. The authors argue that the relationship between safety learning and REM is robust because safety signal learning reflects a positive emotion rather than a negative emotion (e.g., fear), which has been more typically studied in fearpotentiated startle paradigms.

Another recently published study examined REM sleep consolidation and fear inhibition (Datta and O'Malley, 2013). Safety signal and extinction learning are both forms of fear inhibition in which an individual learns that a stimulus does not predict, or no longer predicts, the US. Datta and O'Malley (2013) found that individual differences in physiological events during REM were critical in predicting successful extinction memory consolidation. Although rats showed sim- ilar acquisition and successful reduction of contextual fear during extinction training, approximately half of the extinguished rats showed a marked recovery of fear the next day. Only successful extinguishers showed increased phasic pontine-wave (Pwave) REM activity in the brainstem after extinction, whereas P-wave activity was reduced in those showing impaired extinction retention. Importantly, in both Datta and O'Malley (2013) and Marshall et al. (2014), successful consolidation of the CS- association was predicted by increased REM activity. Prior work by Datta and colleagues (2008) provides insight into the neural mechanisms mediating this relationship. P-wave-generating cells have direct projections to the dorsal hippocampus, and activation of these cells increases glutamate release and elevates mRNA and protein levels of several plasticity-related proteins in the hippocampus and amygdala. These findings provide a neurobiological mechanism by which increased REM sleep activity could facilitate safety signal and extinction consolidation.

Does fragmented REM sleep after a traumatic experience influence an individual's risk of developing PTSD? Not everyone who is exposed to trauma develops PTSD, therefore it is important to understand factors associated with vulnerability or resilience when responding to trauma. Resilience is the ability to adapt and cope despite threatening or challenging situations and it is associated with the resumption of normality following excessive stress (Agaibi and Wilson, 2005). While poor sleep may contribute to the development and progression of PTSD, improving the quantity and quality of sleep (particularly REM) may increase resilience and protect against the development of PTSD when confronted with trauma. Conversely, impaired sleep before trauma may limit one's capacity to manage stressors, thus reducing resilience and increasing the likelihood of PTSD (Bryant et al., 2010). Impaired sleep may (1) limit the cognitive resources available to manage stress, (2) contribute to hyperarousal, (3) represent an additional stressor that compounds the effect of the environmental stressor, or (4) limit restorative sleep that is required to manage stressful events (Bryant et al., 2010). If consolidated REM has a role to play in increasing resilience, studying those at high risk of being exposed to trauma (e.g., emergency service workers and military personnel) is an important avenue for investigation. Indeed, improving sleep in these cohorts may increase resilience and decrease the chance of experiencing future PTSD. Critically, many in these roles are shiftworkers, a group likely to suffer from a persistent high rate of psychological and physical effects of stress and sleep disorders (Edéll-Gustafsson et al., 2002).

Sleep influences on resilience may also have an important role in the treatment of PTSD. While Marshall and colleagues (2014) have demonstrated a role for changes in sleep measures following a fearpotentiated startle procedure in healthy participants, whether the same happens in a population of PTSD sufferers is uncertain. Given that PTSD is associated with nightmares, which may disrupt REM sleep, it is possible that fractured REM may result from PTSD (Germain, 2013). If so, a targeted treatment of sleep to consolidate REM may improve the retention of threat-safety discrimination components of exposure-based cognitive behavioral therapies and accelerate recovery from PTSD. For such an assertion, both prospective and longitudinal studies in those classified as high risk are needed to determine how changes in sleep contribute to increased risk of poor psychiatric outcomes (Germain, 2013). There is a particular need to explore the extent to which sleep disturbances precede and/or result from trauma exposure and/or the onset of PTSD, whether the nature of the sleep disturbance changes over time, and whether sleep interventions are useful treatments for PTSD.

In summary, Marshall et al. (2014) reported that overnight REM sleep was related to improved safety learning, and their findings suggest that REM fragmentation may have a mechanistic role in PTSD via impaired threat-safety discrimination. Their paper provides a translational model for the study of learning and sleep paradigms in humans and suggests new avenues for investigation of issues related to the prevention, development, and treatment of PTSD.

\section{References}

Agaibi CE, Wilson JP (2005) Trauma, PTSD, and resilience: a review of the literature. Trauma Violence Abuse 6:195-216. CrossRef Medline

Briscione MA, Jovanovic T, Norrholm SD (2014) Conditioned fear associated phenotypes as robust, translational indices of trauma-, stressor-, and anxiety-related behaviors. Front Psychiatry 5:88. CrossRef Medline

Bryant RA, Creamer M, O'Donnell M, Silove D, McFarlane AC (2010) Sleep disturbance immediately prior to trauma predicts subsequent psychiatric disorder. Sleep 33:69-74. Medline

Datta S, O’Malley MW (2013) Fear extinction 
memory consolidation requires potentiation of pontine-wave activity during REM sleep. J Neurosci 33:4561-4569. CrossRef Medline

Datta S, Li G, Auerbach S (2008) Activation of phasic pontine-wave generator in the rat: a mechanism for expression of plasticityrelated genes and proteins in the dorsal hippocampus and amygdala. Eur J Neurosci 27: 1876-1892. CrossRef Medline

Edéll-Gustafsson UM, Kritz EI, Bogren IK (2002) Self-reported sleep quality, strain and health in relation to perceived working conditions in females. Scand J Caring Sci 16:179-187. CrossRef Medline

Fu J, Li P, Ouyang X, Gu C, Song Z, Gao J, Han L, Feng S, Tian S, Hu B (2007) Rapid eye movement sleep deprivation selectively impairs recall of fear extinction in hippocampusindependent tasks in rats. Neuroscience 144: 1186-1192. CrossRef Medline

Germain A (2013) Sleep disturbances as the hallmark of PTSD: where are we now? Am J Psychiatry 170:372-382. CrossRef Medline

Germain A, James J, Insana S, Herringa RJ, Mammen O, Price J, Nofzinger E (2013) A win- dow into the invisible wound of war: functional neuroimaging of REM sleep in returning combat veterans with PTSD. Psychiatry Res 211:176-179. CrossRef Medline

Jovanovic T, Kazama A, Bachevalier J, Davis M (2012) Impaired safety signal learning may be a biomarker of PTSD. Neuropharmacology 62:695-704. CrossRef Medline

Marshall AJ, Acheson DT, Risbrough VB, Straus LD, Drummond SP (2014) Fear conditioning, safety learning, and sleep in humans. J Neurosci 34:11754-11760. CrossRef Medline 